18. Kimura, T., et al. 2003. High-density lipoprotein stimulates endothelial cell migration and survival through sphingosine 1-phosphate and its receptors. Arterioscler. Thromb. Vasc. Biol. 23:1283-1288.

19. Gong, M., et al. 2003. HDL-associated estradiol stimulates endothelial NO synthase and vasodilation in an SR-BI-dependent manner. J. Clin. Invest. 111:1579-1587. doi:10.1172/JCI200316777.

20. Lantin-Hermoso, R.L., et al. 1997. Estrogen acutely stimulates nitric oxide synthase activity in fetal pulmonary artery endothelium. Am. J. Physiol. 273:L119-L126.

21. Caulin-Glaser, T., Garcia-Cardena, G., Sarrel, P., Sessa, W.C., and Bender, J.R. 1997. 17 beta-estradiol regulation of human endothelial cell basal nitric oxide release, independent of cytosolic Ca2+ mobilization. Circ. Res. 81:885-892.

22. Shaul, P.W. 2002. Regulation of endothelial nitric oxide synthase: location, location, location. Annu. Rev. Physiol. 64:749-774.
23. Mineo, C., Yuhanna, I.S., Quon, M.J., and Shaul, P.W. 2003. HDL-induced eNOS activation is mediated by Akt and MAP kinases. J. Biol. Chem. 278:9142-9149.

24. Zeiher, A.M., Schachlinger, V., Hohnloser, S.H., Saurbier, B., and Just, H. 1994. Coronary atherosclerotic wall thickening and vascular reactivity in humans. Elevated high-density lipoprotein levels ameliorate abnormal vasoconstriction in early atherosclerosis. Circulation. 89:2525-2532.

25. Li, X.P., et al. 2000. Protective effect of high density lipoprotein on endothelium-dependent vasodilatation. Int. J. Cardiol. 73:231-236.

26. Bisoendial, R.J., et al. 2003. Restoration of endothelial function by increasing high-density lipoprotein in subjects with isolated low highdensity lipoprotein. Circulation. 107:2944-2948.

27. Spieker, L.E., et al. 2002. High-density lipoprotein restores endothelial function in hypercholesterolemic men. Circulation. 105:1399-1402.
28. Kuvin, J.T., et al. 2002. A novel mechanism for the beneficial vascular effects of high-density lipoprotein cholesterol: enhanced vasorelaxation and increased endothelial nitric oxide synthase expression. Am. Heart J. 144:165-172.

29. Ramet, M.E., et al. 2003. High-density lipoprotein increases the abundance of eNOS protein in human vascular endothelial cells by increasing its half-life. J. Am. Coll. Cardiol. 41:2288-2297.

30. Blair, A., Shaul, P.W., Yuhanna, I.S., Conrad, P.A., and Smart, E.J. 1999. Oxidized low density lipoprotein displaces endothelial nitric-oxide synthase (eNOS) from plasmalemmal caveolae and impairs eNOS activation. J. Biol. Chem. 274:32512-32519.

31. Uittenbogaard, A., Shaul, P.W., Yuhanna, I.S. Blair, A., and Smart, E.J. 2000. High density lipoprotein prevents oxidized low density lipoprotein-induced inhibition of endothelial nitric-oxide synthase localization and activation in caveolae. J. Biol. Chem. 275:11278-11283.

\title{
Fighting cancer by disrupting C-terminal methylation of signaling proteins
}

\author{
Steven Clarke ${ }^{1,2}$ and Fuyuhiko Tamanoi ${ }^{2,3}$ \\ ${ }^{1}$ Department of Chemistry and Biochemistry, \\ ${ }^{2}$ Molecular Biology Institute, and \\ ${ }^{3}$ Department of Microbiology, Immunology, and Molecular Genetics, Jonsson \\ Comprehensive Cancer Center, University of California, Los Angeles, California, USA
}

\begin{abstract}
Protein methylation at the C-terminus of mammalian isoprenylated proteins has been implicated in membrane attachment, protein-protein interactions, and protein stability. A new paper describes surprising results: in the absence of methylation some target proteins have increased stability, whereas others have decreased stability. The decreased stability of the RhoA protein is correlated with an increased resistance to Ras-dependent transformation and suggests the basis for the development of a new approach to antitumor therapy (see the related article beginning on page 539).
\end{abstract}

\section{J. Clin. Invest. 113:513-515 (2004). doi:10.1172/JCI200421059.}

Covalent modification of proteins facilitates a tremendous expansion of their functional potential. Some modifications serve to enlarge the chemical diversity of proteins beyond that provided by the 20 standard amino acids utilized in protein synthesis, providing the new shapes and reactive groups that allow new types of binding and catalytic interactions. Other modifications, often reversible, serve to modulate protein function. Among the large

\footnotetext{
Address correspondence to: Steven Clarke, 640 Boyer Hall, University of California, Los Angeles, California 90095-1569, USA. Phone: (310) 825-8754; Fax: (310) 825-1968; E-mail: clarke@mbi.ucla.edu.

Conflict of interest: The authors have declared that no conflict of interest exists. Nonstandard abbreviations used: isoprenylcysteine carboxyl methyltransferase (Icmt); S-adenosylhomocysteine (AdoHcy).
}

number of reactions that modify intracellular eucaryotic proteins are three sequential enzymatic steps that recognize proteins synthesized with a C-terminal CAAX tetrapeptide motif, where $\mathrm{C}$ is a cysteine residue, $\mathrm{A}$ is generally an aliphatic residue, and $X$ can be a variety of residues (1-3). Such proteins are initially lipidated in a reaction that adds either a 15-carbon farnesyl or a 20 -carbon geranylgeranyl group to the sulfur atom of the cysteine residue. This step is followed by the removal of the three AAX amino acids generating a C-terminal isoprenylcysteine residue. The third step is a potentially reversible methyl esterification reaction of the newly exposed C-terminal cysteinyl carboxyl group by a membranebound enzyme of the endoplasmic reticulum (Figure 1). Many proteins are modified in this way, including some nuclear lamins, a cGMP phosphodiesterase, and several small Gproteins involved in cell signaling, such as the Ras and Rho proteins. The importance of Ras, especially the activated oncogenic forms, has led to a large interest in this pathway in terms of the possibility of controlling human cancers.

These reactions can provide the modified protein increased membrane attachment with the newly formed hydrophobic C-terminus (4), directed binding to signaling partners via the isoprenyl and methyl groups $(5,6)$, and protection against proteolytic degradation $(7,8)$. In this issue of the JCI, Bergo et al. provide new insight into these functions and have identified new targets for the development of anticancer drugs (9). In this study, they developed transgenic mice (and fibroblast cell lines derived from these mice) where the expression of the Icmt gene encoding isoprenylcysteine carboxyl methyltransferase (Icmt) can be controlled by the Cre-loxP system. There are two striking results from this study. First, the authors show that loss of the methyltransferase can have different effects in different methylaccepting proteins. For example, they show that Ras proteins are stabilized but the RhoA protein is destabilized. Second, they show that the decreased levels of RhoA in the absence of methylation results in increased levels of p21 $1^{\text {Cip } 1}$ and the inhibition of the cell cycle. The authors thus present a case for anticancer therapeutic approaches based on inhibiting Icmt. 


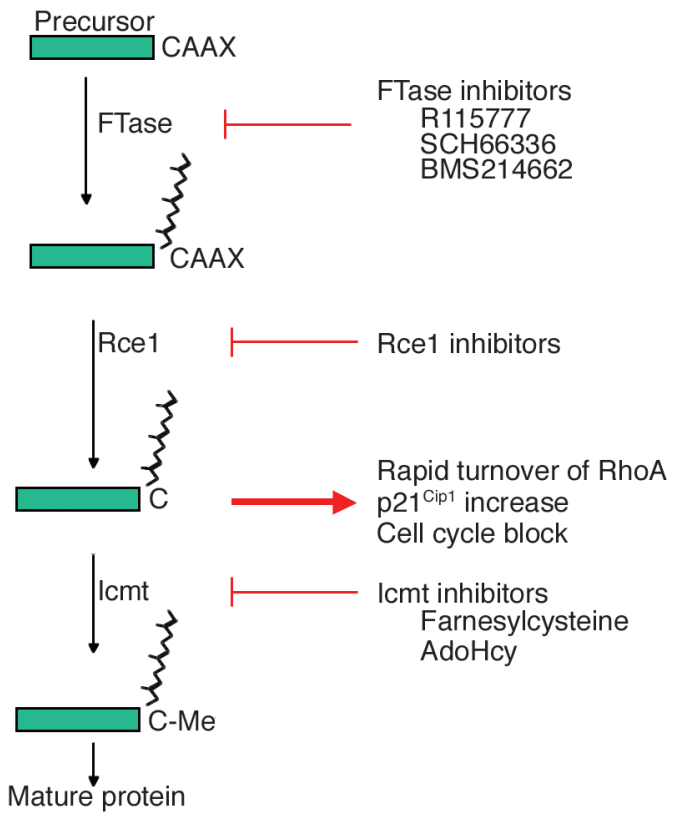

Stabilization of isoprenylated proteins by methyl ester formation Unmethylated isoprenylated cysteine groups at the C-terminus of a protein can be particularly susceptible to proteolytic attack. For example, in yeast strains lacking the STE14 gene encoding the ortholog of the mammalian Icmt gene, a soluble form of the Ras protein accumulates and has the gel mobility of the non-isoprenylated precursor protein rather than the unmethylated protein $(7,10)$. Unmethylated intracellular yeast a-mating factor is subject to a fivefold higher rate of proteolytic degradation (11). In mammalian cells, inhibition of methylation in a macrophage cell line results in the decrease of the half-life of the RhoA small G-protein from 31 to 12 hours, and of the Cdc 42 small G-protein from 15 to 11 hours (8).

Biochemists search for simplicity in understanding metabolic reactions, and it was thus reassuring to see that Bergo et al. found that the level of RhoA was reduced 90 to $95 \%$ in Icmtdeficient fibroblasts (9), clearly confirming the results of Backlund (8) in a distinct cell culture system. In fact the complete absence of Icmt in the fibroblasts led to an even greater reduction in the half-life of RhoA from 22 to 2.8 hours. However, nature often reveals her more complex side, and Bergo et al. found that another isoprenylated signaling protein dis- played just the opposite behavior! They measured an increase in the halflife of K-Ras from 13.9 hours to 32.5 hours (9). Clearly, there is now no direct relationship between proteolytic stability and the presence of the carboxyl terminal methyl ester.

\section{Loss of methyl esterification by the Icmt enzyme is associated with cell growth inhibition}

Bergo et al. found that inhibition of the methyltransferase caused reduced growth and inhibited K-Ras-induced oncogenic transformation (9). While this result is exciting, it is perhaps not totally unexpected, given that K-Ras is a substrate for isoprenylation and methylation. What was surprising, however, was the authors' finding that the effect was apparently not directly associated with the inhibition of K-Ras function itself; Ras-dependent growth factor-induced activation of Erk and Akt was unaffected. It turns out that much of the inhibition of transformation can be laid at the door of unmethylated RhoA, where the reduction in protein level is correlated with an upregulation of the $\mathrm{p} 21^{\text {Cip } 1}$ protein that binds cyclins and stops the cell cycle. Furthermore, the effect of Icmt deficiency is not limited to K-Rasinduced transformation: transformation induced by an activated (V599E) form of B-Raf was also attenuated by the loss of Icmt.

\section{Figure 1}

Modification pathway of proteins for isoprenylation/methylation and effects of inhibitors of each enzymatic step. Proteins ending with the CAAX motif undergo farnesylation in the cytosol. Subsequent removal of three C-terminal amino acids and C-terminal methylation are catalyzed by the endoplasmic reticulum-bound enzymes Rce 1 and Icmt, respectively. Inhibition of Icmt leads to rapid turnover of RhoA, increase of p 21 Cip1, and cell cycle block. R115777, SCH66336, and BMS214662 are farnesyltransferase (Ftase) inhibitors, while farnesylcysteine and AdoHcy inhibit Icmt.

\section{Signaling from the endoplasmic reticulum}

The finding by Bergo et al. (9) that K-Ras can signal activation of downstream events - even though they show that $\mathrm{K}$-Ras is not associated with the plasma membrane - is quite intriguing. What could account for this observation? It is possible that a small amount of K-Ras is associated with the plasma membrane and is enough to signal downstream events. It is also possible that a Ras-independent pathway functions to catalyze growth factor-induced activation of Erk and Akt in the absence of Icmt. Finally, Bergo et al. (9) suggest the possibility that K-Ras can signal from intracellular locations such as endoplasmic reticulum and Golgi. This idea is particularly interesting, given the recent report that Ras targeted to the intracellular membranes can activate the Erk pathway (12).

\section{Cancer therapeutics}

Inhibition of posttranslational modification of signaling proteins provides a promising approach to anticancer therapy. Enzymes catalyzing the three consecutive biochemical reactions on CAAX motif proteins are all potential targets of drug action. Much of the recent work has focused on small-molecule inhibitors of the protein farnesyltransferase that catalyzes the addition of a C-15 isoprenyl group to the cysteine side chain of many CAAX motif-containing proteins (13). Many of these 
compounds are competitive inhibitors of farnesyltransferase with respect to its substrates farnesyl pyrophosphate and the CAAX motif $(1,14)$. In addition, compounds that inhibit farnesyltransferase by chelating zinc, a tightly associated metal that is involved in catalysis, have been identified (15). In preclinical studies, these compounds could inhibit the growth of tumors in mice and even cause regression of Ha-Ras-activated tumors (14). Several inhibitors are currently being evaluated in clinical trials, and beneficial effects have been reported with hematological and solid malignancies (16). However, it has been disappointing that these inhibitors have so far failed to exhibit efficacy against pancreatic tumors where oncogenic forms of Ras are found at high frequency (16). This failure has been attributed to the action of the apparently redundant geranylgeranyltransferase I on K-Ras.

The mixed success with farnesyltransferase inhibitors has led investigators to explore the possibility of targeting the protease Rce1, which removes the AAX residues, as well as the Icmt enzyme. Since only a single enzyme catalyzes each of these reactions $(17,18)$, this approach may be more effective. It has been shown, for example, that Rce1 inactivation reduces Ras-induced transformation (19). The results of Bergo et al. (9) now provide convincing evidence that targeting Icmt can also be effective in blocking the transforming potential of $\mathrm{K}$-Ras and other isoprenylated proteins.

\section{Present status of inhibitors of Icmt and other methyltransferases}

Two approaches have been taken to develop inhibitors of cellular methylation reactions. The first is based on the inhibition of nearly all methyltransferases by the product $S$-adenosylhomocysteine (AdoHcy) (20). Compounds that increase cellular AdoHcy levels by inactivating AdoHcy hydrolase have both antiviral (21) and antitumor (22) activity. The targets of these drugs are not clear; it is possible that their effects may occur on what has been estimated as more than 300 methyltransferases in the human genome (23) as well as at non-methyltransferase sites (21). But one target may certainly be Icmt. Along those lines, inhibition of Icmt through elevation of AdoHcy levels has been pro- posed as a mechanism for the antiproliferative effects of methotrexate, a widely used chemotherapeutic agent (24).

A second approach has been to develop inhibitors that would be specific to Icmt (reviewed in refs. 3 and 7). There was initial excitement about the possibility of using derivatives of farnesylcysteine to this end, but this enthusiasm was tempered by the realization that these compounds could block proteinprotein interactions based on isoprene group recognition (25). Additionally, incubation of Ha-Ras-transformed rat embryo fibroblasts with farnesylcysteine under conditions that resulted in the $60-70 \%$ reduction in Ha-Ras methylation did not significantly affect the growth or transformation of these cells (26). However, given the results of Bergo et al. (9), it now appears worthwhile to resume the search for more effective inhibitors of the Icmt-encoded methyltransferase that may mimic the antiproliferative effect of the knockout of this enzyme in mice. Because the Icmt enzyme is one of a relatively small class of methyltransferases characterized by multiple membrane-spanning segments, it may be possible to develop a new class of inhibitors that take advantage of this particular structure (3).

\section{Acknowledgments}

Work in the authors' laboratories is supported by NIH grants GM26020 and AG18000 to Steven Clarke, and CA41996 and CA32737 to Fuyuhiko Tamanoi.

1. Sattler, I., and Tamanoi, F. 1996. Prenylation of Ras and inhibitors of prenyltransferases. In Regulation of the RAS signaling network. H. Maruta and A.W. Burgess, editors. R.G. Landes. Austin, Texas, USA. 95- 137.

2. Sinensky, M. 2000. Recent advances in the study of prenylated proteins. Biochim. Biophys. Acta. 1484:93-106.

3. Young, S.G., Ambroziak, P., Kim, E., and Clarke, S. 2001. Postisoprenylation protein processing CXXX (CaaX) endoproteases and isoprenylcysteine carboxyl methyltransferase. In The enzymes. 3rd edition. Volume XXI, Protein lipidation. F. Tamanoi and D.S. Sigman, editors. Academic Press. San Diego, California, USA. 155-213.

4. Magee, A.I., and Seabra, M.C. 2003. Are prenyl groups sticky fingers or greasy handles? Biochem.J. 376:3-4.

5. Dietrich, A., et al. 2003. Studies on G-protein alpha-beta-gamma heterotrimer formation reveal a putative S-prenyl-binding site in the alpha subunit. Biochem. J. 376:449-456.

6. Chen, F., Ng, P.S., Faull, K.F., and Lee, R.H. 2003 Cone photoreceptor betagamma-transducin: posttranslational modification and interaction with phosducin. Invest. Ophthalmol. Vis. Sci. 44:4622-4629.
7. Hrycyna, C.A., and Clarke, S. 1993. Modification of eukaryotic signalling proteins by C-terminal methylation reactions. Pharmacol. Ther. 59:281-300.

8. Backlund, P.S., Jr. 1997. Post-translational processing of RhoA: carboxyl methylation of the carboxyl-terminal prenylcysteine increases the halflife of RhoA. J. Biol. Chem. 272:33175-33180.

9. Bergo, M.O., et al. 2004. Inactivation of Icmt inhibits transformation by oncogenic K-Ras and B-Raf. J. Clin. Invest. 113:539-550. doi:10.1172/ JCI200418829.

10. Fujiyama, A., Matsumoto, K., and Tamanoi, F. 1987. A novel yeast mutant defective in the processing of ras proteins: assessment of the effect of the mutation on processing steps. EMBO J. 6:223-228.

11. Sapperstein, S., Berkower, C., and Michaelis, S. 1994. Nucleotide sequence of the yeast STE14 gene, which encodes farnesylcysteine carboxyl methyltransferase, and demonstration of its essential role in a-factor export. Mol. Cell. Biol. 14:1438-1449.

12. Chiu, V.K., et al. 2002. Ras signalling on the endoplasmic reticulum and the Golgi. Nat. Cell Biol. 4:354-350.

13. Tamanoi, F., Gau, C.L., Jiang, C., Edamatsu, H., and Kato-Stankiewicz, J. 2001. Protein farnesylation in mammalian cells: effects of farnesylation inhibitors on cancer cells. Cell. Mol. Life Sci. 58:1636-1649.

14. Gibbs, J.B. 2001. Farnesyltransferase inhibitors. In The enzymes. 3rd edition. Volume XXI, Protein lipidation. F. Tamanoi and D.S. Sigman, editors. Academic Press. San Diego, California, USA. 81-103.

15. Hamasaki, A., Naka, H., Tamanoi, F., Umezawa K., and Otsuka, M. 2003. A novel metal-chelating inhibitor of protein farnesyltransferase. Bioorg. Med. Chem. Lett. 13:1523-1526.

16. Brunner, T.B., et al. 2003. Farnesyltransferase inhibitors: an overview of the results of preclinical and clinical investigations. Cancer Res. 63:5656-5668

17. Otto, J.C., Kim, E., Young, S.G. and Casey, P.J. 1999. Cloning and characterization of a mammalian prenyl protein-specific protease. J. Biol. Chem. 274:8379-8382.

18. Bergo, M.O., et al. 2001. Isoprenylcysteine carboxyl methyltransferase deficiency in mice. J. Biol. Chem. 276:5841-5845.

19. Bergo, M.O., et al. 2002. Absence of the CAAX endoprotease Rce1: effects on cell growth and transformation. Mol. Cell. Biol. 22:171-181.

20. Clarke, S., and Banfield, K. 2001. S-adenosylmethionine-dependent methyltransferases: potential targets in homocysteine-linked pathology. In Homocysteine in health and disease. R. Carmel and D. Jacobsen, editors. Cambridge University Press. Cambridge, United Kingdom. 63-78.

21. Gordon, R.K., et al. 2003. Anti-HIV-1 activity of 3 deaza-adenosine analogs. Inhibition of S-adenosylhomocysteine hydrolase and nucleotide congeners. Eur. J. Biochem. 270:3507-3517.

22. Wnuk, S.F. 1997. Anticancer and antiviral effects and inactivation of S-adenosyl-L-homocysteine hydrolase with $5^{\prime}$-carboxaldehydes and oximes synthesized from adenosine and sugar-modified analogues. J. Med. Chem. 40:1608-1618.

23. Katz, J.E., Dlakic, M., and Clarke, S. 2003. Automated identification of putative methyltransferases from genomic open reading frames. $\mathrm{Mol}$. Cell. Proteomics. 2:525-540.

24. Winter-Vann, A.M., et al. 2003. Targeting Ras signaling through inhibition of carboxyl methylation: An unexpected property of methotrexate. Proc. Natl. Acad. Sci. U. S. A. 100:6529-6534.

25. Scheer, A., and Gierschik, P. 1993. Farnesylcysteine analogs inhibit chemotactic peptide receptor-mediated G-protein activation in human HL-60 granulocyte membranes. FEBS Lett. 319:110-114.

26. Volker, C., et al. 1991. Effects of farnesylcysteine analogs on protein carboxyl methylation and signal transduction. J. Biol. Chem. 266:21515-21522. 